\author{
Military Technical College \\ Kobry El-Kobbah, \\ Cairo, Egypt.
}

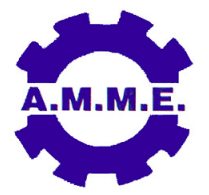

\title{
CRACK DETECTION IN BEAMS USING AXIAL AND TRANSVERSE NATURAL FREQUENCIES
}

\author{
M. M. Mostafa ${ }^{1}$, M. Tawfik ${ }^{2}$ and H. M. Negm ${ }^{3}$
}

\begin{abstract}
Crack detection in structures has attracted the researchers for many years. Several crack detection attempts use measurements of the structure's modal parameters, like the natural frequencies and mode shapes. One of The easiest parameter among them to measure and correlate to cracks is the natural frequencies. Several approaches focused on crack detection in beams and bars using the changes in the natural frequencies. The direct problem has been addressed, but the inverse problem is a challenging one, where it starts by measuring certain structural properties and estimate the crack location and size. In most research works, the natural frequencies of the intact structure or historical measurements have to be known in advance. Another feature in similar studies is to handle the beam action and bar action separately. The inverse problem of an Euler-Bernoulli beam with an open crack is addressed here. Unlike these models, the present model utilizes the first axial and transverse natural frequencies to determine the size and location of the crack, without prior historical measurements.
\end{abstract}

\section{KEY WORDS}

Structural Health Monitoring, Crack Detection, Modal Analysis, Euler-Bernoulli Beam.

1 Ph.D. Student, Aerospace Engineering Department, Cairo University, Giza, Egypt, Email: maladdin@yahoo.com.

2 Assistant Professor, Aerospace Engineering Department, Cairo University, Giza, Egypt. Email: mohammad.tawfik@gmail.com.

3 Professor of aircraft structures, Aerospace Engineering Department, Cairo University, Giza, Egypt. 


\section{INTRODUCTION}

Successful early detection of cracks can help avoid structural failure which could cause dangerous accidents [1]. This stimulated many researchers to focus on Structural Health Monitoring (SHM) in the last three decades. In the forward problem, the researcher attempts to correlate changes in certain system parameters to known damage properties. The inverse problem, which is more realistic, is to start by measuring some of the system parameters and then try to quantify the damage without prior knowledge of its characteristics. Published reviews of SHM discuss several methods, theories and approaches in the field. Modal analysis is one important method among them. It relies on measuring structural modal parameters (e.g. natural frequencies, mode shapes...etc), then relates their values to the structure health.

Sekhar [2] and Adewuyi et al. [3] present different vibration-based damage identification methods. Fan and Qiao [4] presented classification for vibration-based damage techniques along with a brief discussion on each class.

Although beams and bars are simple in shape and analysis, they are important structural elements. Understanding their behavior can pave the way towards understanding more complex structures. This continues to draw the attention of researchers to beams and bars.

Friswell and Penny [5] investigated open crack modeling techniques. They concluded that simple open crack models can simulate cracks effectively in the low frequency range. One of the widely used crack modeling techniques is to divide the beam into two segments connected by springs [6, 8, 9], as shown in Fig 1 . This technique is both simple and efficient.

Fernandez-Saez et al. [6] provide an approximate method to calculate the fundamental frequency of a cracked simply supported Euler-Bernoulli beam.

Sinha et al. [7] proposed a simplified analysis to detect open crack properties based on updating a FE model using measured natural frequencies. Lin et al. [8] used Transfer Matrix analysis to estimate the first four natural frequencies and the corresponding mode shapes for Euler-Bernoulli beams with an arbitrary number of cracks. Patil and Maiti [9] proposed an approach to solve the inverse problem of beams with multiple cracks based on a Transfer Matrix approach similar to that presented by Lin et al [8] In Patil and Maiti investigation, an influence matrix is evaluated through measuring the changes in natural frequencies to quantify the cracks. Liang et al. [10] proposed a numerical technique to evaluate multiple cracks in a beam based on the natural frequencies changes, similar to Patil and Maiti investigation [9]. Chen et al. [11] extended Liang et al. [10] analysis utilizing the Dynamic Mesh-Refinement Method for an Euler-Bernoulli beam.

Crack detection in bars, rods and shafts is particularly important as they are used in all rotating machines and gearboxes, and may lead to tragic accidents [1]. Ruotolo and Surace [14] derived expressions for the natural frequencies of bars with multiple cracks. Dilena and Morassi [18] developed a numerical model to detect damage in 
rods using natural and anti-resonant frequencies. Chondros et al. [19, 20] derived the governing differential equation of a cracked bar.

In the present study, the inverse problem of cracked Euler-Bernoulli beams will be addressed using the change in the natural frequencies. As crack quantification requires evaluating its location and depth, at least two measurements are needed i.e. two or more natural frequencies. If a crack is located near a nodal point, the natural frequency will not exhibit any change regardless of the crack severity. Accordingly, more modes are needed to avoid this drawback. Available studies in the literature analyze beam and bar problems separately. While the difference between a bar and a beam is the loading direction. However, axial and transverse modes will continue to be structural properties regardless of the loading condition. In this analysis, unlike other approaches, a mixture of axial and transverse natural frequencies will be used to quantify the crack. The two measurements needed to quantify a single crack will be the first axial and transverse natural frequencies measured for the cracked beam. A major advantage of the present approach is that it neither requires prior knowledge of the natural frequencies of the intact beam nor any historical measurements.

\section{MATHEMATICAL MODEL}

The starting point of the analysis is the analytical solution of a cracked beam in both axial and transverse directions. The crack is simulated by axial and rotational massless springs. Several boundary conditions will be examined. These conditions are Fixed-Free, Fixed-Roller, Fixed-Pinned, Pinned-Pinned and Pinned-Roller support conditions. The procedure holds for any other boundary conditions.

\section{Model Assumptions}

The cracked beam of concern, as shown in Fig. 1, has the following properties:

- Beam is made of an isotropic linear elastic material.

- The beam is a uniform, slender, Euler-Bernoulli beam, which implies that the slope of the elastic line is the first derivative of the transverse displacement.

- The beam contains a single double edge open crack.

- The crack will be represented by massless axial and rotational springs to introduce displacement discontinuity in the axial direction and slope discontinuity in the transverse direction.

- The change in mass properties due to the crack is negligible.

As shown in Fig. 1, the length of the beam is $L$, and the crack is located a distance $\alpha L$ from the left end of the beam, where $\alpha$ ranges from 0 to 1 . The ratio of total crack depth to the beam thickness is $(\delta)$. The problem will be divided into separate bar and beam problem.

\section{Bar Problem}

The bar is divided into two segments at the crack location. The spatial distribution of the displacement field of the bar is given by [14]: 


$$
\begin{aligned}
& u_{1}(x)=A \cos (\lambda x)+B \sin (\lambda x) \\
& u_{2}(x)=C \cos (\lambda x)+D \sin (\lambda x)
\end{aligned}
$$

where $u_{1}$ and $u_{2}$ are the axial displacement of the left and right segments of the bar respectively. The wave number $\lambda$ is related to the cracked bar natural frequency by [14]:

$$
\lambda=\omega \sqrt{\frac{\rho}{E}}
$$

where $\rho, E$ and $\omega$ are the mass density, modulus of elasticity and the natural frequency of the bar respectively. The constants $A, B, C$ and $D$ can be evaluated by applying the boundary and compatibility conditions.

From the axial motion perspective, the boundary conditions for Fixed-Free are the same for Fixed-Roller, and Pinned-Roller conditions. They are given by:

$$
u_{1}(0)=0=A, \quad u_{2}^{\prime}(L)=0
$$

Gives:

$$
u_{1}(x)=B \sin (\lambda x), \quad u_{2}^{\prime}(L)=\lambda[-C \sin (\lambda L)+D \cos (\lambda L)]=0
$$

The compatibility conditions at the crack location imply that the derivative (representing axial strain) from both sides is the same, while the force is proportional to the axial spring stiffness:

$$
\begin{gathered}
u_{1}^{\prime}(\alpha L)=u_{2}^{\prime}(\alpha L) \\
u_{1}^{\prime}(\alpha L)=\frac{K_{a}}{E A}\left[u_{2}(\alpha L)-u_{1}(\alpha L)\right]
\end{gathered}
$$

where $A$ is the cross section area and $K_{a}$ is the axial spring stiffness. This stiffness is a function of both the material properties and crack depth ratio, and will be discussed in a later section.

Applying the condition given by equation (5), we get:

$$
\lambda B \cos (\alpha \lambda L)=\lambda C[-\sin (\alpha \lambda L)+\tan (\lambda L) \cos (\alpha \lambda L)]
$$

Applying the condition given by (6):

$$
\lambda B \cos (\alpha \lambda L)=\frac{K_{a}}{E A}[C[\cos (\alpha \lambda L)+\tan (\lambda L) \sin (\alpha \lambda L)]-B \sin (\alpha \lambda L)]
$$

Solving equations (7) and (8) for $K_{a}$ :

$$
\frac{K_{a}}{E A}=\lambda \cos ^{2}(\alpha \lambda L)[\tan (\lambda L)-\tan (\alpha \lambda L)]
$$

As the spring stiffness is function of the crack depth, as will be described in later section, the expression given by (9) gives the relation between the crack properties, 
in terms of location and depth, and the natural frequency of the cracked bar. This expression is valid for the Fixed-Free, Fixed-Roller, and Pinned-Roller beams.

For Fixed-Pinned and Pinned-Pinned beams, the boundary conditions are given by:

$$
u_{1}(0)=0=A, \quad u_{2}(L)=0
$$

Gives:

$$
u_{1}(x)=B \sin (\lambda x), \quad u_{2}(L)=[-C \cos (\lambda L)+D \sin (\lambda L)]=0
$$

Applying the compatibility conditions given by (5) and (6), and solving the resulting equations for the spring stiffness gives:

$$
\frac{K_{a}}{E A}=-\frac{\lambda}{2 \sin (\lambda L)}[\cos (\lambda L)+\cos (\lambda L(1-2 \alpha))]
$$

\section{Beam Problem}

For the beam problem, similar to analysis of bar problem, the crack is represented by a rotational spring and analytical solution will be assumed for each beam segment. The beam properties are identical to those stated above.

The spatial distribution of the transverse displacement of the beam segments to the left and right of the crack are given below. Equations are cast in matrix form for ease of manipulations:

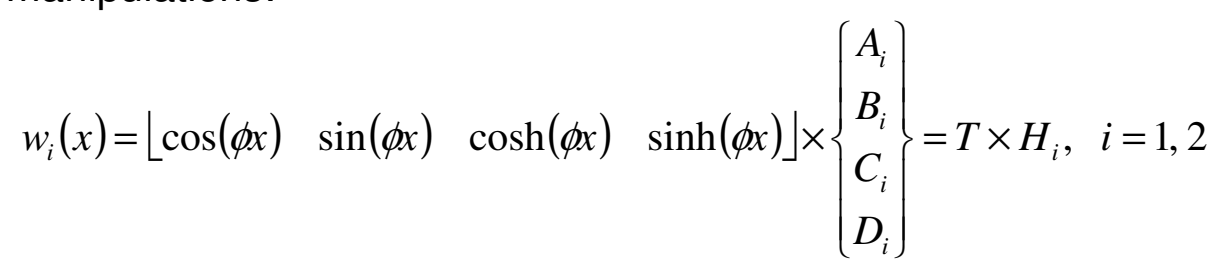

where $\phi$ in case of beam is given by [[9] ]:

$$
\phi^{4}=\left(\omega^{2} \frac{\rho A}{E I}\right)
$$

where $A$ and $I$ are the cross section area and second moment of area respectively. The boundary conditions for the above mentioned cases as follows:

For Fixed-Free beam:

$$
w_{1}(0)=0, \quad w_{1}^{\prime}(0)=0, \quad w_{2}^{\prime \prime}(L)=0, \quad w_{2}^{\prime \prime \prime}(L)=0
$$

For Pinned-Pinned and Pinned-Roller beam:

$$
w_{1}(0)=0, \quad w_{1}^{\prime \prime}(0)=0, \quad w_{2}(L)=0, \quad w_{2}^{\prime \prime}(L)=0
$$

For Fixed-Roller and Fixed-Pinned beam: 


$$
w_{1}(0)=0, \quad w_{1}^{\prime}(0)=0, \quad w_{2}(L)=0, \quad w_{2}^{\prime \prime}(L)=0
$$

The compatibility conditions for all cases are the same and given by [[9] ]:

$$
\begin{aligned}
& w_{1}(\alpha L)=w_{2}(\alpha L), \quad w_{1}^{\prime \prime}(\alpha L)=w_{2}^{\prime \prime}(\alpha L), \quad w_{1}^{\prime \prime \prime}(\alpha L)=w_{2}^{\prime \prime \prime}(\alpha L), \\
& \text { and } \\
& w_{1}^{\prime \prime}(\alpha L)=\frac{K_{r}}{E I}\left[w_{2}^{\prime}(\alpha L)-w_{1}^{\prime}(\alpha L)\right]
\end{aligned}
$$

where $K_{r}$ is the rotational spring stiffness at the crack.

Each set of the above boundary conditions with the compatibility conditions are cast in a homogenous matrix form as detailed in Table 1: Equations used to estimate a crack. For the four boundary conditions and the first three compatibility conditions, the wave number $\phi$ appears as a common factor with powers corresponding to the degree of differentiation. Since it has a positive non-zero value, it will be crossed out from these equations.

For Fixed-Free beam, and to get a nonzero solution of equation (26) in the appendix, the determinant of the coefficient matrix must vanish, which leads to the following solution for $K_{r}$ :

$$
\frac{K_{r}}{E I}=\phi \frac{\left(\begin{array}{l}
2 C(\beta) \operatorname{Sh}(\beta)-2 C h(\beta) S(\beta)+S(\phi L)[C h(\phi L)+C h(\phi L(2 \alpha-1))] \\
-S h(\phi L)[C(\phi L(2 \alpha-1))+C(\phi L)]+2 S h(\phi L(\alpha-1))[C(\phi L(\alpha-1))-S(\phi L(\alpha-1))]
\end{array}\right)}{4(C(\phi L) C h(\phi L)+1)}
$$

where $C, S, C h$ and $S h$ in equations (26) and (19) stand for cosine, sine, hyper cosine and hyper sine functions respectively, and $\beta$ is given by:

$$
\beta=\alpha \phi L
$$

The expression above matches the one derived by Patil and Maiti [9].

Similarly, for the Pinned-Pinned and Pinned Roller beam problems, the same procedure is utilized. To derive a similar relation to (19), the boundary conditions given by (16) are used. The nonzero solution of equation (27) in the appendix is obtained when the determinant of the coefficient matrix vanishes, which leads to the following solution for $K_{r}$ :

$$
\frac{K_{r}}{E I}=\frac{\phi}{4}\left(\frac{C(\phi L(1-2 \alpha))-C(\phi L)}{S(\phi L)}+\frac{C h(\phi L(1-2 \alpha))-C h(\phi L)}{S h(\phi L)}\right)
$$

Again, the expression above matches the one derived by Patil and Maiti [9].

For Fixed-Roller and Fixed-Pinned beams, using the boundary conditions given by (17) and solving equation (28), an expression for $K_{r}$ is given by: 


$$
\frac{K_{r}}{E I}=\frac{\phi}{4}\left(\begin{array}{l}
C(\phi L) C h(\phi L(1-2 \alpha))-C(\phi L(1-2 \alpha)) C h(\phi L)+2 S(\phi L) \operatorname{Sh}(\phi L)+ \\
\frac{S(\phi L) \operatorname{Sh}(\phi L(1-2 \alpha))+S(\phi L(1-2 \alpha)) \operatorname{Sh}(\phi L)+4 S(\phi L(1-\alpha)) \operatorname{Sh}(\phi L(1-\alpha))}{C(\phi L) \operatorname{Sh}(\phi L)-C h(\phi L) S(\phi L)}
\end{array}\right)
$$

\section{Spring Stiffness-Crack Relation}

Several relations between the spring stiffness and crack depth are found in the literature. These relations are based on fracture mechanics. They differ based on the crack shape. The relations given below are among the most widely used. For a double edge open crack, according to Ruotolo and Surace [14], the axial spring stiffness is given by:

$$
K_{a}^{-1}=\frac{2 h\left(1-v^{2}\right)}{E A} S(\delta)
$$

where $h, E, A$ and $v$ are the section height, material modulus of elasticity, cross section area and Poisson's ratio respectively. $S$ is a function of the crack depth ratio $\delta$ as follows:

$$
S(\delta)=0.713 \delta^{8}-1.0368 \delta^{7}+0.5803 \delta^{6}+1.2055 \delta^{5}-1.0368 \delta^{4}+0.2381 \delta^{3}+0.9852 \delta^{2}
$$

The rotational spring stiffness that simulates a double edge open crack, according to Haisty and Springer [22], is defined by:

$$
K_{r}^{-1}=\frac{9 \pi \delta^{2}}{b\left(\frac{h}{2}\right)^{2} E}\left(0.5033-0.9022 \delta+3.412 \delta^{2}-3.181 \delta^{3}+5.793 \delta^{4}\right)
$$

where $b$ is the section width.

Similar expressions are available in the literature for single edge cracks. The present analysis holds to be true for other spring expressions.

\section{PROPOSED SOLUTION}

The solution procedure will be briefly described for a given crack located at distance $\alpha L$ from the left side, where $\alpha$ ranges between 0.1 and 0.9 . The crack depth to section height ratio is given by $\delta$. A procedure is required to detect the crack location and depth using measurement of the longitudinal and transverse natural frequencies. The procedure can be summarized as follows:

1. For the cracked beam, measure the first axial and transverse natural frequencies.

2. According to the beam boundary conditions, select the two appropriate equations derived for both axial and transverse modes as per Table 1 below.

3. Substitute the natural frequencies obtained in step (1) in the right hand sides of the corresponding equations. 
4. Equate the spring stiffness calculated by the equations selected in step (2) to those given by equations (23) and (25) with the help of equation (24).

5. Solve the resulting coupled system of equations for the crack depth and crack location.

\section{CASE STUDIES}

To test the proposed procedure, the axial and transverse natural frequencies of cracked beam are needed. Although, results on cracked bars and cracked beams are published in literature, they are not given for the same test cases. Thus, FE model was constructed assuming crack location and depth to calculate the axial and transverse natural frequencies for the same case study. The FE model was verified for bar and beam separately using those on literature [5, 14] ensuring robust results of the FE model.

According to the proposed approach, for a cracked beam, to locate the crack and estimate the crack depth, the first axial and transverse natural frequencies need to be measured. Due to absence of the measured data, the finite element method is used. So first, we introduce a known crack depth and location to the FE model to estimate the corresponding axial and transverse natural frequencies. These two natural frequencies are used as input to the equations given in Table 1. Solving the equations with the natural frequencies are known, one can obtain the corresponding crack location and depth. The error is calculated by comparing the estimated crack depth and location (output of the equations given in Table 1) with those used to estimate the natural frequencies in the FEM (assumed crack values).

However, these equations are highly non-linear in both crack location $\alpha$ and crack depth $\delta$. Thus an iterative numerical technique is required to solve these two equations to obtain the crack properties. Newton-Raphson method is utilized to evaluate the crack properties.

The following numerical examples are used to test the proposed procedure. The beam is assumed to be of length $L$ of $0.8 \mathrm{~m}$ and section width of $0.02 \mathrm{~m}$ and height of $0.06 \mathrm{~m}$. The material mass density $\rho$ equals $7800 \mathrm{~kg} / \mathrm{m}^{3}$, modulus of elasticity $E$ equals $210 \mathrm{GPa}$, and Poisson's ratio $v$ equals 0.3 .

\section{Fixed-Free Beam}

The location of the assumed crack ranges from $0.1 \mathrm{~m}(12.5 \%)$ to $0.7 \mathrm{~m}(87.5 \%)$ along the $0.8 \mathrm{~m}$ beam, and the crack depth ratio ranges from $10 \%$ to $90 \%$. The solution using the proposed approach gives small errors in most cases. The error in estimating the location does not exceed 4\%, while the error in the depth does not exceed $10 \%$ in most cases, as shown in Fig. 2 and Fig. 3. The accuracy of the crack quantification of the proposed technique is affected by the accuracy natural frequency measurements, or estimation in case of FEM model. Thus some readings are significantly lower in accuracy such as crack of $60 \%$ in depth at $87.5 \%$ of the beam length. See Fig. 3 below. 


\section{Fixed-Roller Beam}

For Fixed-Roller beam the crack location and depth ranges are as stated above. Fig. 4 and Fig. 5 show the error in crack estimation for different crack depth and location combinations. The error is generally small except for cracks located near the fixed support. Also, as indicated above, accuracy of the natural frequency estimation affects the crack prediction accuracy.

\section{Fixed-Pinned Beam}

Results of the Fixed-Roller beam are shown in Fig. 6 and Fig. 7. The proposed method failed to detect the crack located at $50 \%$ of the beam regardless of the crack depth. Apart from this and some limited other cases, the error is small.

\section{Pinned-Pinned Beam}

For Pinned-Pinned beam, the results are shown in Fig. 8 and Fig. 9. The crack estimation is highly accurate for this case, with the crack location less accurate than crack depth.

\section{Pinned-Roller Beam}

For Pinned-Roller beam, the errors in crack estimations are shown in Fig. 10 and Fig. 11. Curves show high accuracy in crack location estimation, and with inferior accuracy in crack depth estimation in some cases.

\section{DISCUSSION AND CONCLUSIONS}

The proposed approach is a simple straight forward procedure to solve the inverse problem of crack detection in Euler-Bernoulli beams. The approach can be applied to any set of boundary conditions. In general, estimating the crack properties utilizing this approach gives accurate results in both crack location and depth estimates. However, the error in estimating the crack properties is affected by accuracy of the natural frequency measurements. A large error in the natural frequency results in a large error in crack quantification. When the crack is located near one end, assuming Euler-Bernoulli for the short part is one possible source of inaccuracy of crack prediction. Another source of error is the spring stiffness relations as a function of the crack depth especially at large crack depths. In some cases of boundary conditions crack prediction has bad accuracy, and in a single case the procedure fails to detect the crack, See Fig. 6 and Fig. 7. In some cases, the changes in natural frequencies are so small causing increase in the error of the crack perdition up to values as high as $200 \%$.

Since the Pinned-Roller beam is symmetric about its mid span with respect to the transverse motion, the first natural frequency will be same if the crack is located at the same distance from either support. However, this is not the case for the longitudinal motion, where the bar is treated as a fixed-free bar. That is why other methods which depend on transverse modes only fail to locate the actual crack position in such a case. However, since the axial vibration mode is not symmetric, 
the present approach converges only to the correct crack location regardless of symmetry of the problem in the transverse direction. On the other hand, this approach converges to both symmetric positions if the problem is symmetric in the axial and transverse modes, like the case of Pinned-Pinned beam.

\section{REFERENCES}

[1] Samuel, P. D. and Pines, D. J., "A Review of Vibration-based Techniques for Helicopter Transmission Diagnostics," Journal of Sound and Vibration, Vol. 282, 2005, pp. 475-508.

[2] Sekhar, A. S., "Multiple Cracks Effects and Identification," Mechanical Systems and Signal Processing, Vol. 22, 2008, pp. 845-878.

[3] Adewuyi, A. P., Wu, Z. and Serker, N.H.M., "Assessment of Vibration-based Damage Identification Methods Using Displacement and Distributed Strain Measurements," Structural Health Monitoring, Vol. 8, 2009, pp. 443-461.

[4] Fan, W. and Qiao, P. "Vibration-based Damage Identification Methods: A Review and Comparative Study," Structural Health Monitoring, Vol. 10, 2011, pp. 83-111.

[5] Friswell, M.I. and Penny, J., "Crack Modeling for Structural Health Monitoring," Structural Health Monitoring, Vol. 1, 2002, pp. 139-148.

[6] Fernandez-Saez, J., Rubio, L. and Navarro, C., "Approximate Calculations of the Fundamental Frequency for Bending Vibration of Cracked Beams," Journal of Sound and Vibration, Vol. 225, No. 2, 1999, pp. 345-352.

[7] Sinha, J. K., Friswell, M. I., and Edwards, S., "Simplified Models for the Location of Cracks in Beam Structures using measured Vibration Data," Journal of Sound and Vibration, Vol. 251, No. 1, 2002, pp. 13-38.

[8] Lin, H. P., Chang, S. C., and Wu, J. D., "Beam Vibrations with an Arbitrary Number of Cracks," Journal of Sound and Vibration, Vol. 258, No. 5, 2002, pp. 987-999.

[9] Patil, D. P. and Maiti, S. K., "Detection of Multiple Cracks using frequency Measurements," Engineering Fracture Mechanics, Vol. 70, 2003, pp. 15531572.

[10] Liang, R. Y., Hu, J. and Choy, F., "Quantitative NDE Technique for Assessing Damages in Beam Structures," Journals of Engineering Mechanics, Vol. 118, 1992, 1468-1487. Loya, J.A., Rubio, L. and Fernandez-Saez, J. "Natural frequencies for bending vibrations of Timoshenko cracked beams," Journal of Sound and Vibration, Vol 290, 2006, pp. 640-653.

[11] Chen, X. F., Zi, Y. Y. and He, Z. J., "Identification of Multiple Cracks using a Dynamic Mesh-Refinement Method," The Journal of Strain Analysis for Engineering Design, Vol. 41, No. 1, 2006, pp. 31-41.

[12] Sumant, P.S., and Miati, S. K., "Crack Identification in a Beam Using PZT Sensors," Smart Materials and Structures, Vol. 15, 2006, pp. 695-703.

[13] Guyomar, D., Lallart, M. Monnier, T., Wang, X. and Petit, L., "Passive Impact Location Estimation Using Piezoelectric Sensors," Structural Health Monitoring, Vol. 8, 2009, pp. 357-367.

[14] Ruotolo, R. and Surace, C., "Natural Frequency of a Bar with Multiple Cracks," Journal of Sound and Vibration, Vol. 272, 2004, pp. 301-316. 
[15] Liu, X. and Paurobally, R., "Robust Damage Metric in Terms of Magnitude and Phase for Impedance-based Structural Health Monitoring," Structural Health Monitoring, Vol. 8, 2009, pp. 303-311.

[16] Al-Ajmi, M. A. and Benjeddou, A., "Damage Indication in Smart Structures using Modal Effective Electromechanical Coupling Coefficients," Smart Materials and Structures, Vol. 17, No. 3, 2008, pp. 15.

[17] Bouboulas, AS. and Anifantis, NK., "Finite Element Modeling of Vibrating Beam with a Breathing Crack: Observations on Crack Detection," Structural Health Monitoring, Vol. 10, 2011, pp. 131-145.

[18] Dilena, M. and Morassi, A. "Structural Health Monitoring of Rods Based on Natural Frequency and Antiresonant Frequency Measurements," Structural Health Monitoring, Vol. 8, 2009, pp. 149-173.

[19] Chondros, T. G., Dimarogonas, A. D. and Yao, J., "A Consistent Cracked Bar Vibration Theory," Journal of Sound and Vibration, Vol. 200, No. 3, 1997, pp. 303-313.

[20] Chondros, T. G., Dimarogonas, A. D. and Yao, J., "Longitudinal Vibration of a Continuous Cracked Bar," Engineering Fracture Mechanics, Vol. 61, 1998, pp. 593-606.

[21] Ganeriwala, S. N., Kanakasabai, V., and Richardson, M., "Modes Indicate Cracks in Wind Turbine Blades," Conference Proceedings of the Society for Experimental Mechanics Series, Vol. 8, 2011, pp. 509-513.

[22] Haisty, B. S., and Springer, W. T., "A General Beam Element for Use in Damage Assessment of Complex Structures," Journal of Vibration, Acoustics, Stress, and Reliability in Design, Vol. 110, 1988, pp. 389-394. 
Table 1. Equations used to estimate a crack.

\begin{tabular}{|c|c|c|c|}
\hline \multicolumn{2}{|l|}{$\begin{array}{l}\text { Boundary } \\
\text { Conditions }\end{array}$} & $\begin{array}{l}\text { Axial mode } \\
\text { equation }\end{array}$ & $\begin{array}{l}\text { Transverse mode } \\
\text { equation }\end{array}$ \\
\hline Fixed-Free & 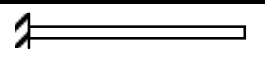 & (9) & (19) \\
\hline Fixed-Roller & $\not$ & (9) & (22) \\
\hline Fixed-Pinned & $\not$ A & $(12)$ & (22) \\
\hline Pinned-Pinned & $\bar{F}$ & (12) & (21) \\
\hline Pinned-Roller & 严 & (9) & (21) \\
\hline
\end{tabular}
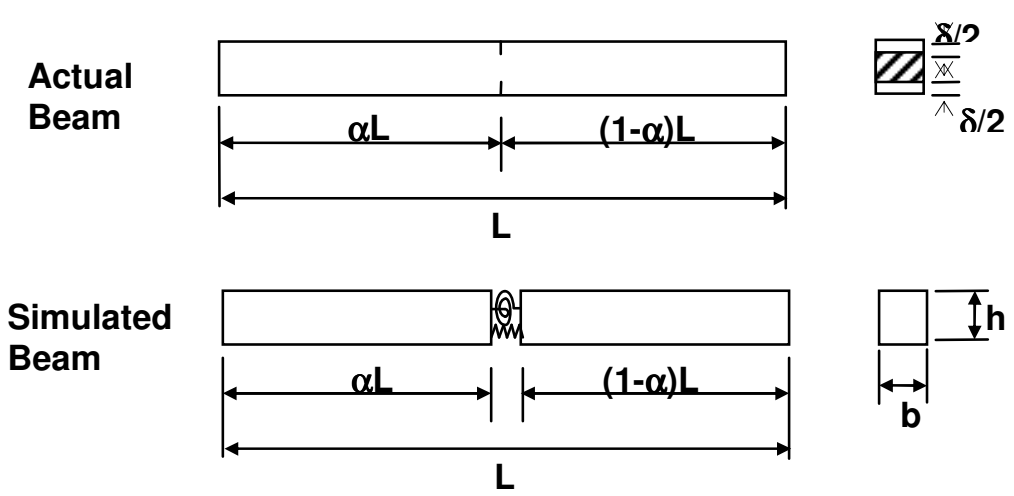

Fig 1. Cracked 1-D Structure.

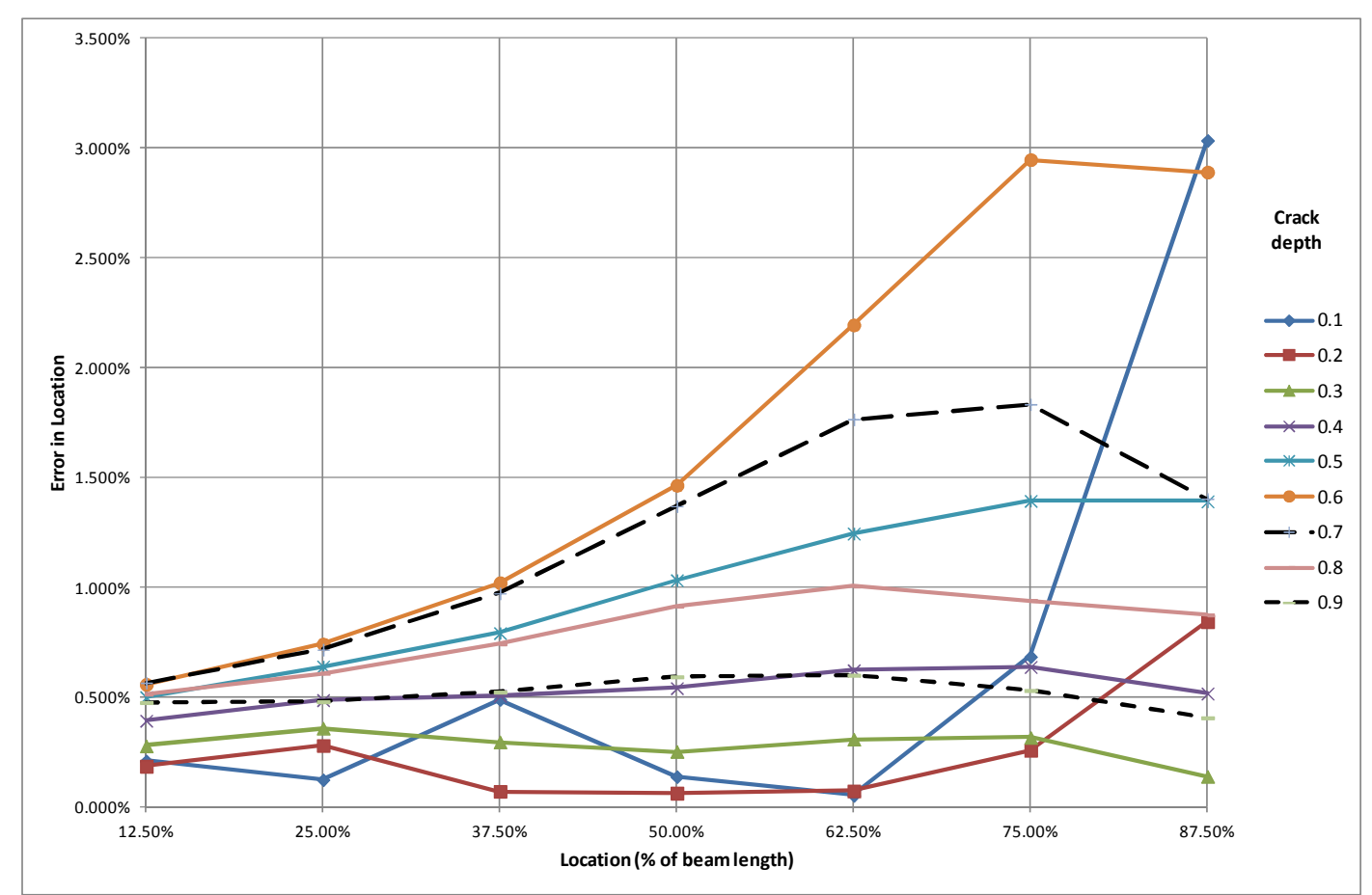

Fig 2. Fixed-Free beam. Error in crack location estimation for differnet crack depths. 


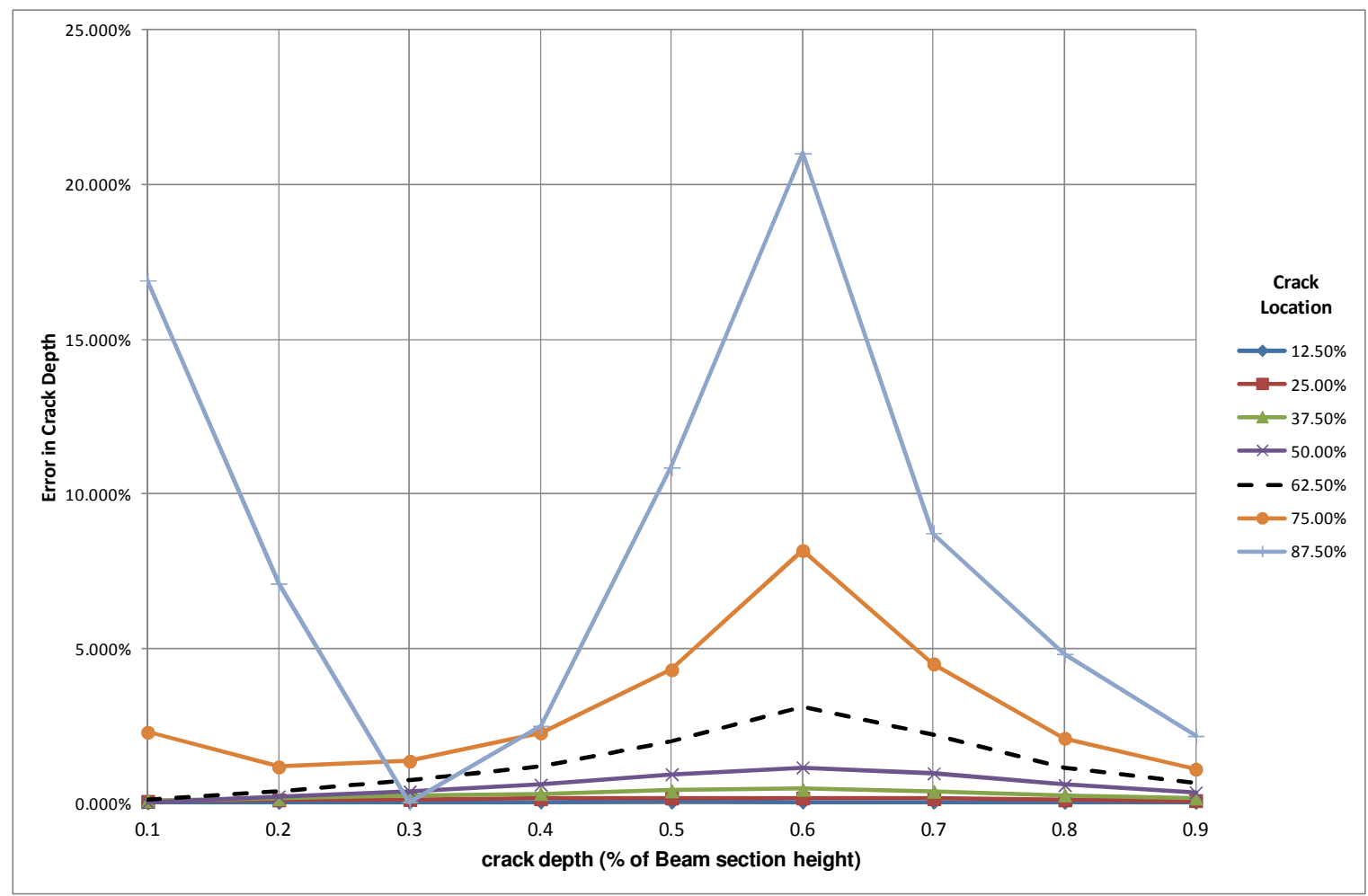

Fig 3. Fixed-Free beam. Error in crack depth estimation for differnet crack locations.

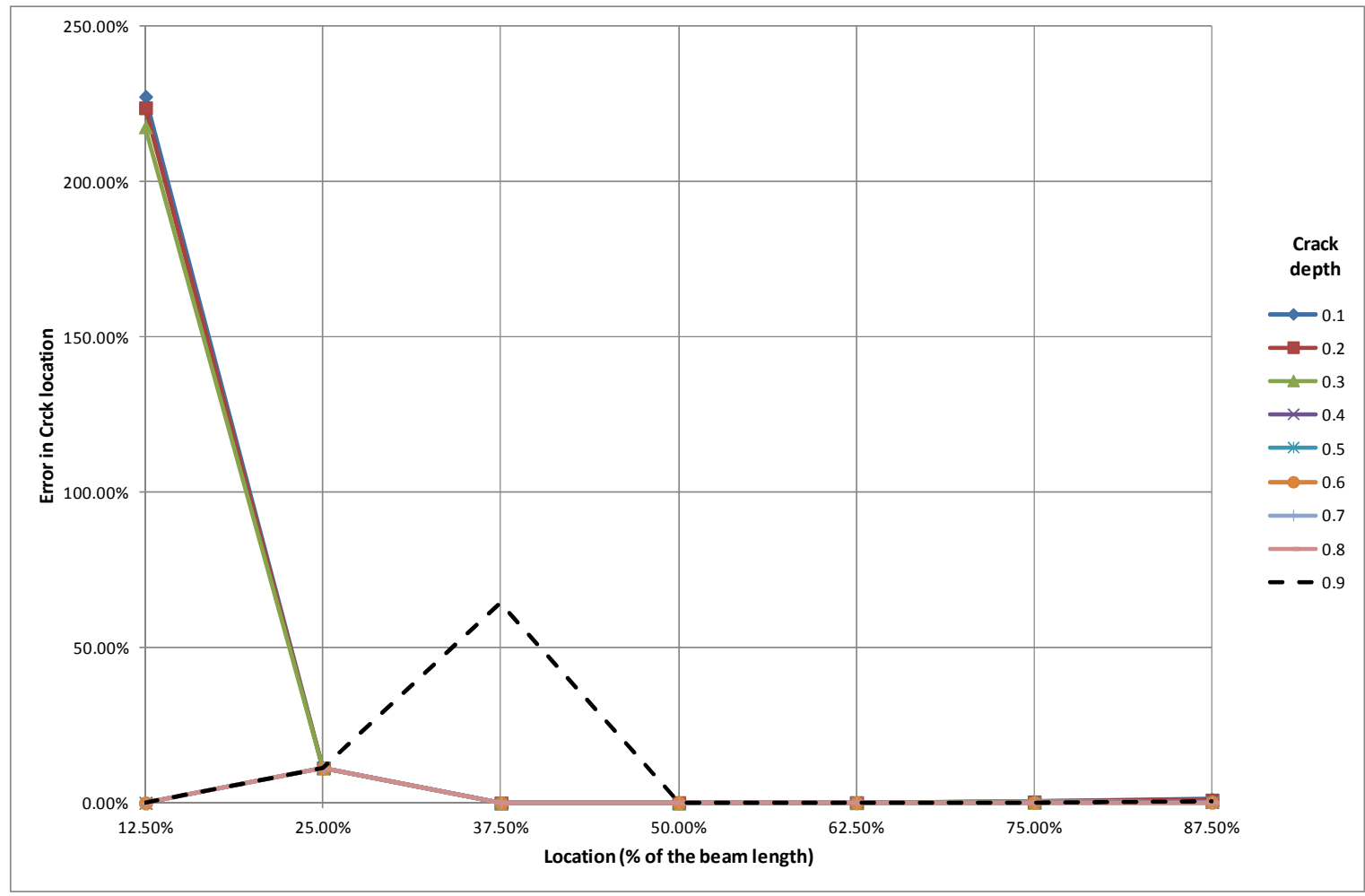

Fig 4. Fixed-Roller: Error in crack location for different crack depths. 


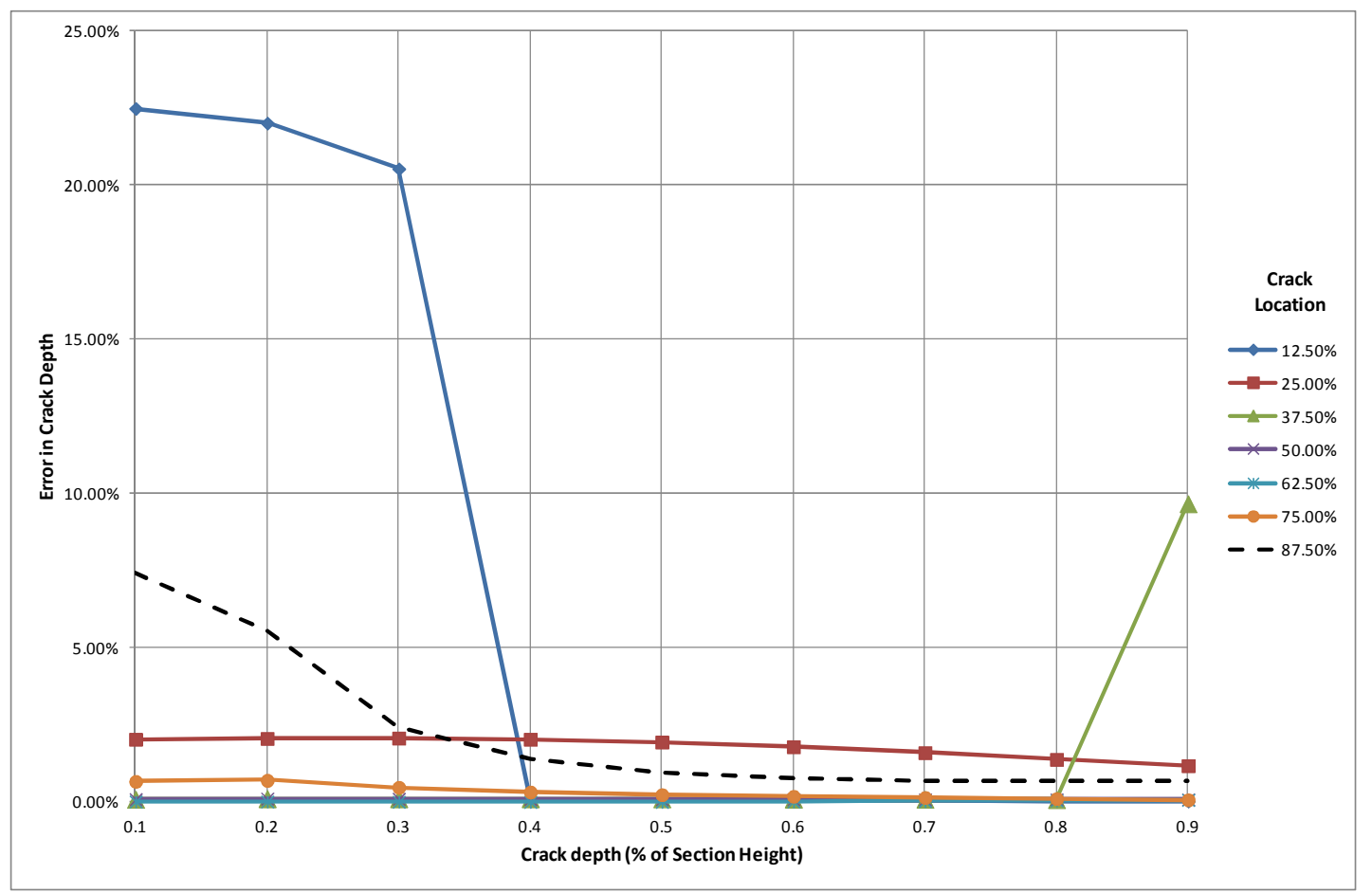

Fig 5. Fixed-Roller: Error in crack depth for different crack locations.

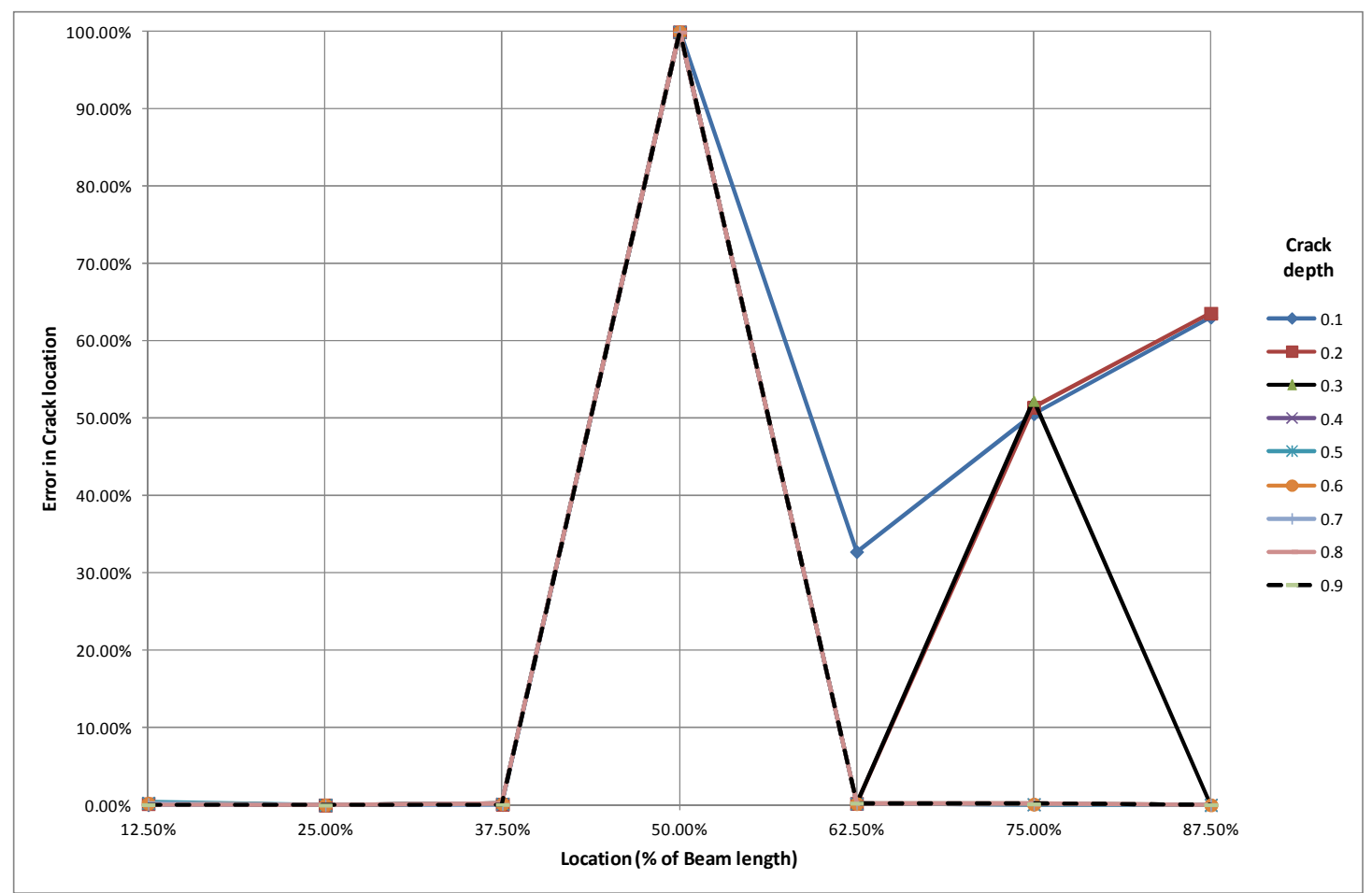

Fig 6. Fixed-Pinned: Error in crack location for different crack depths. 


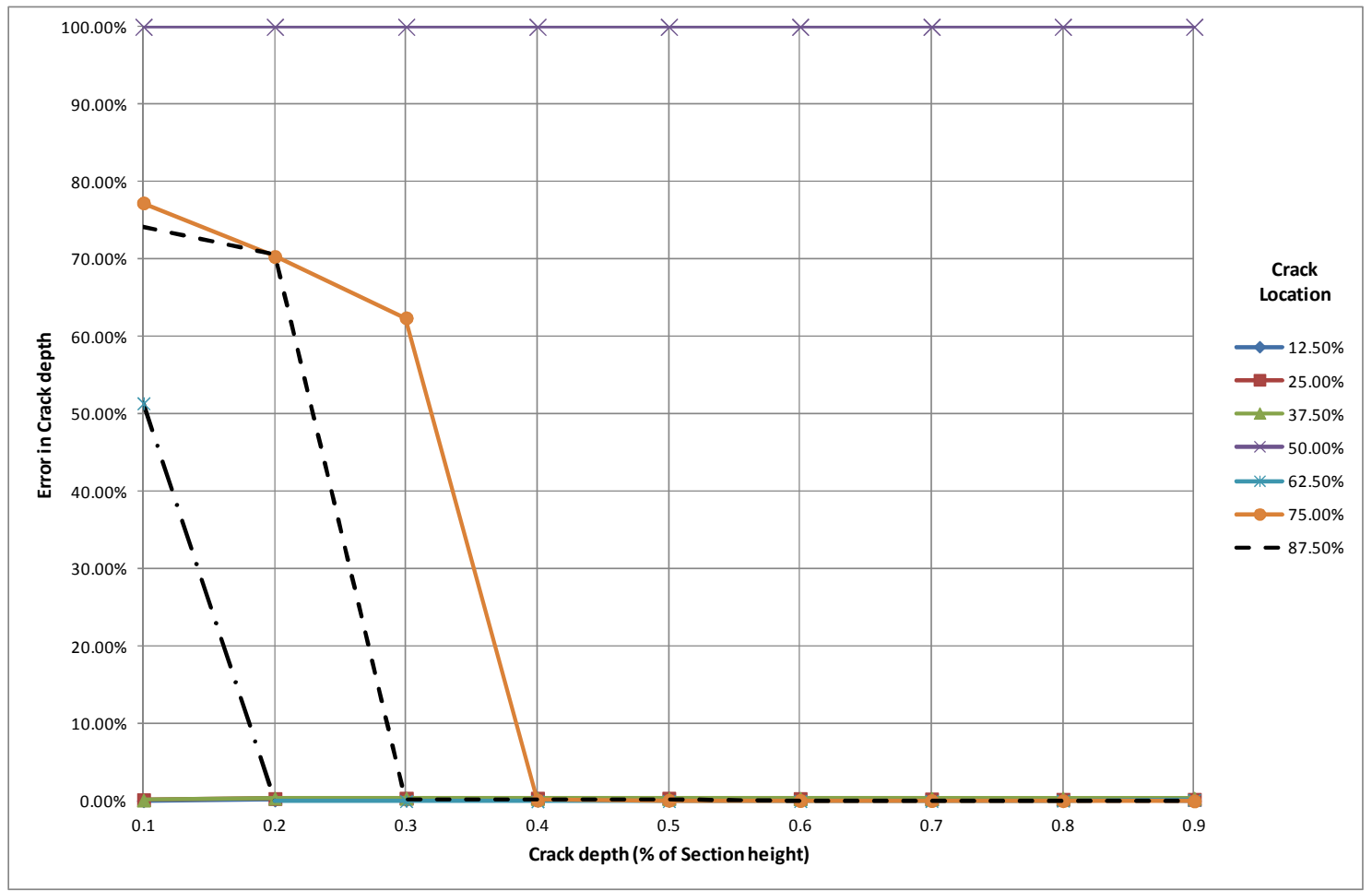

Fig 7. Fixed-Pinned: Error in crack depth for different crack locations.

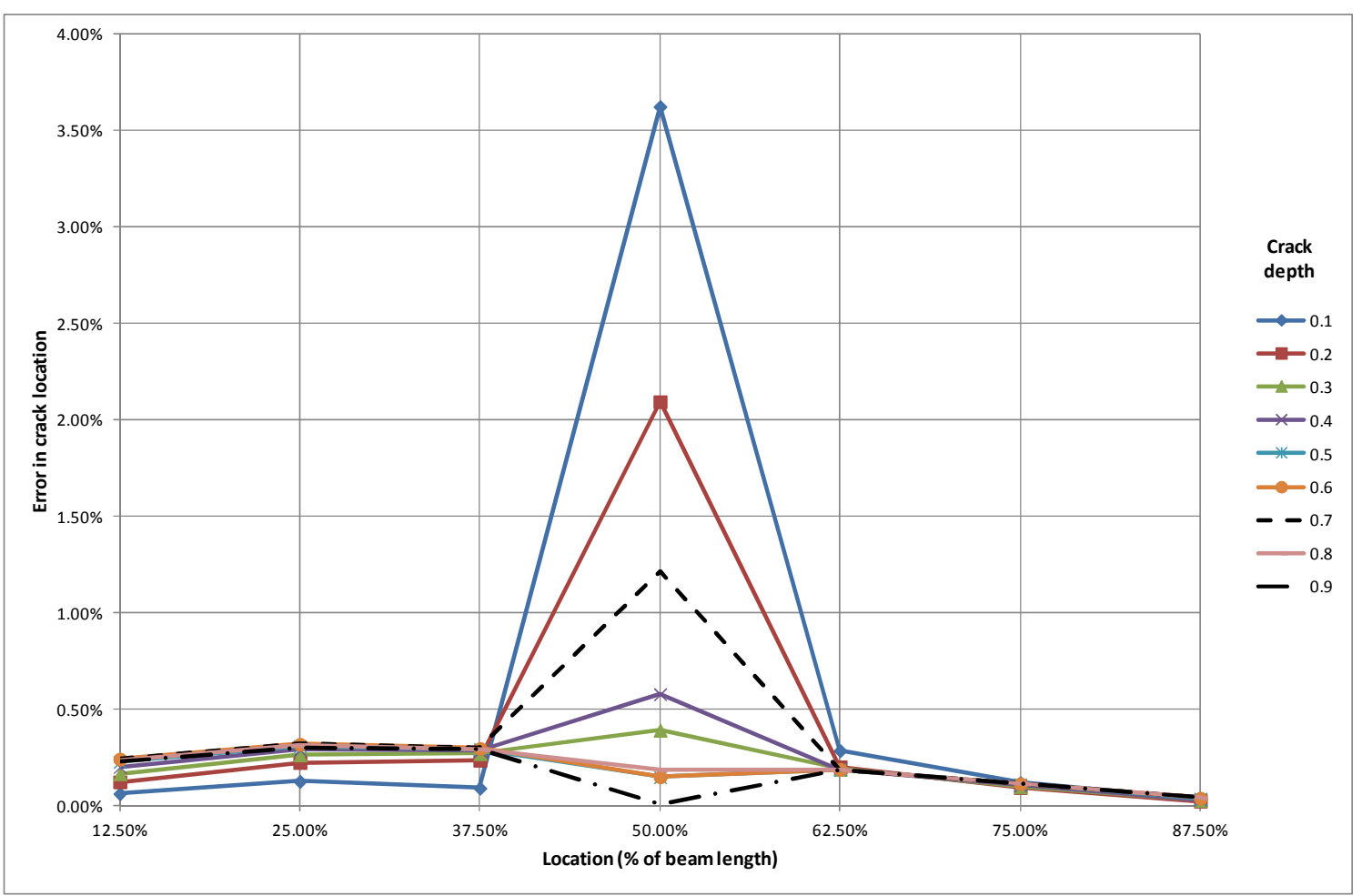

Fig 8. Pinned-Pinned: Error in crack location for different crack depths. 


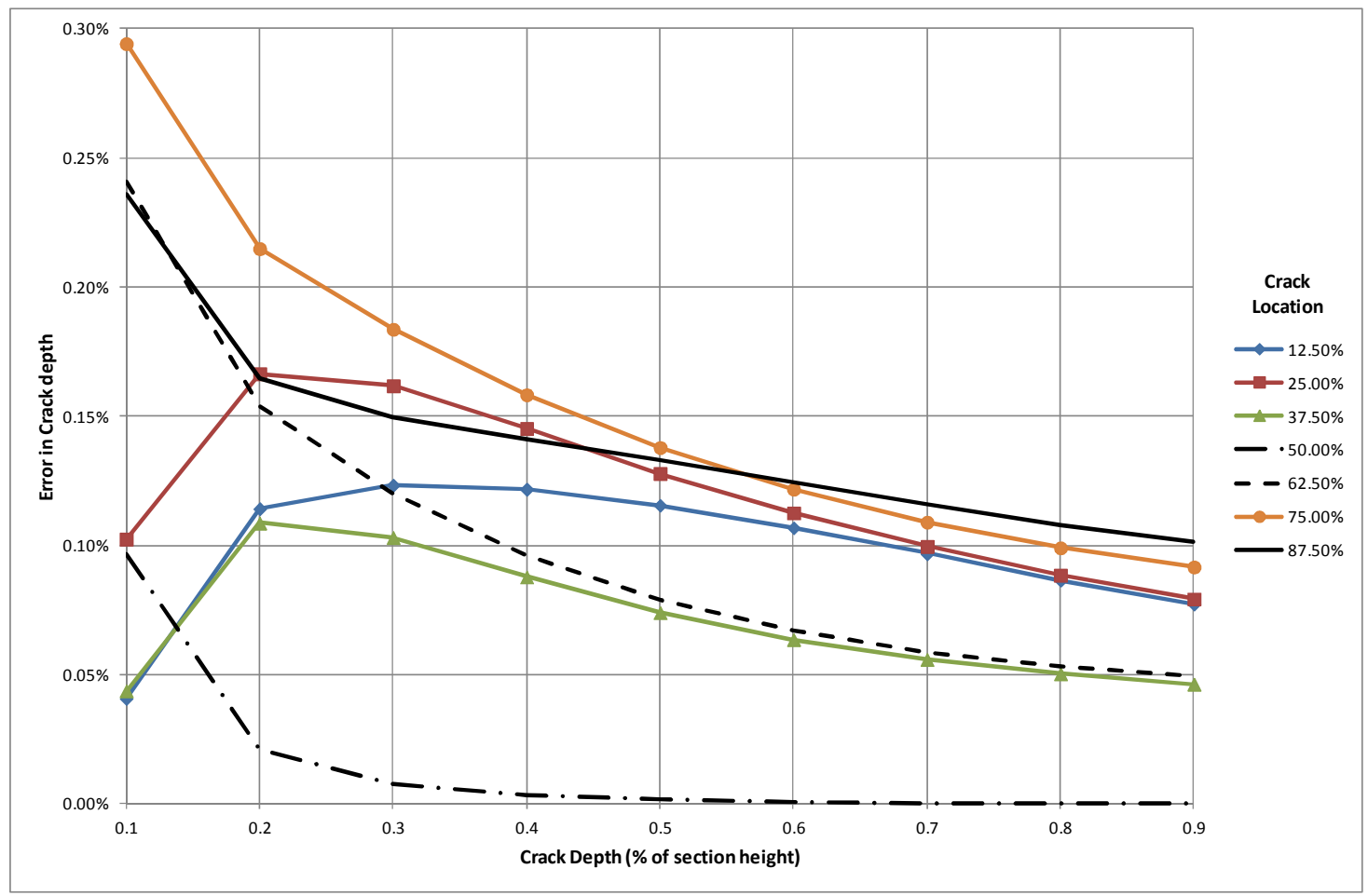

Fig 9. Pinned-Pinned: Error in crack depth for different crack locations.

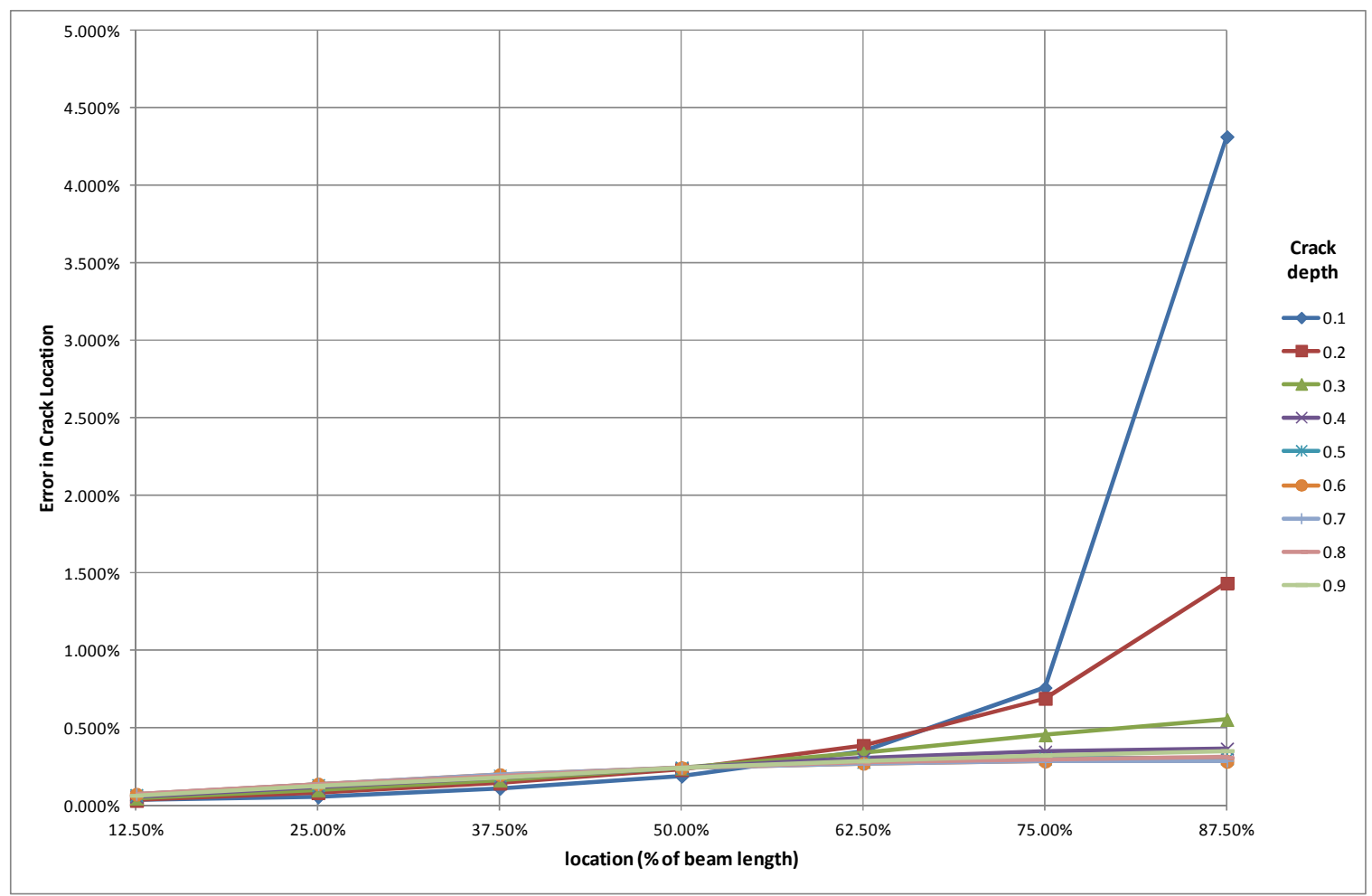

Fig 10. Pinned-Roller: Error in crack location for different crack depths. 


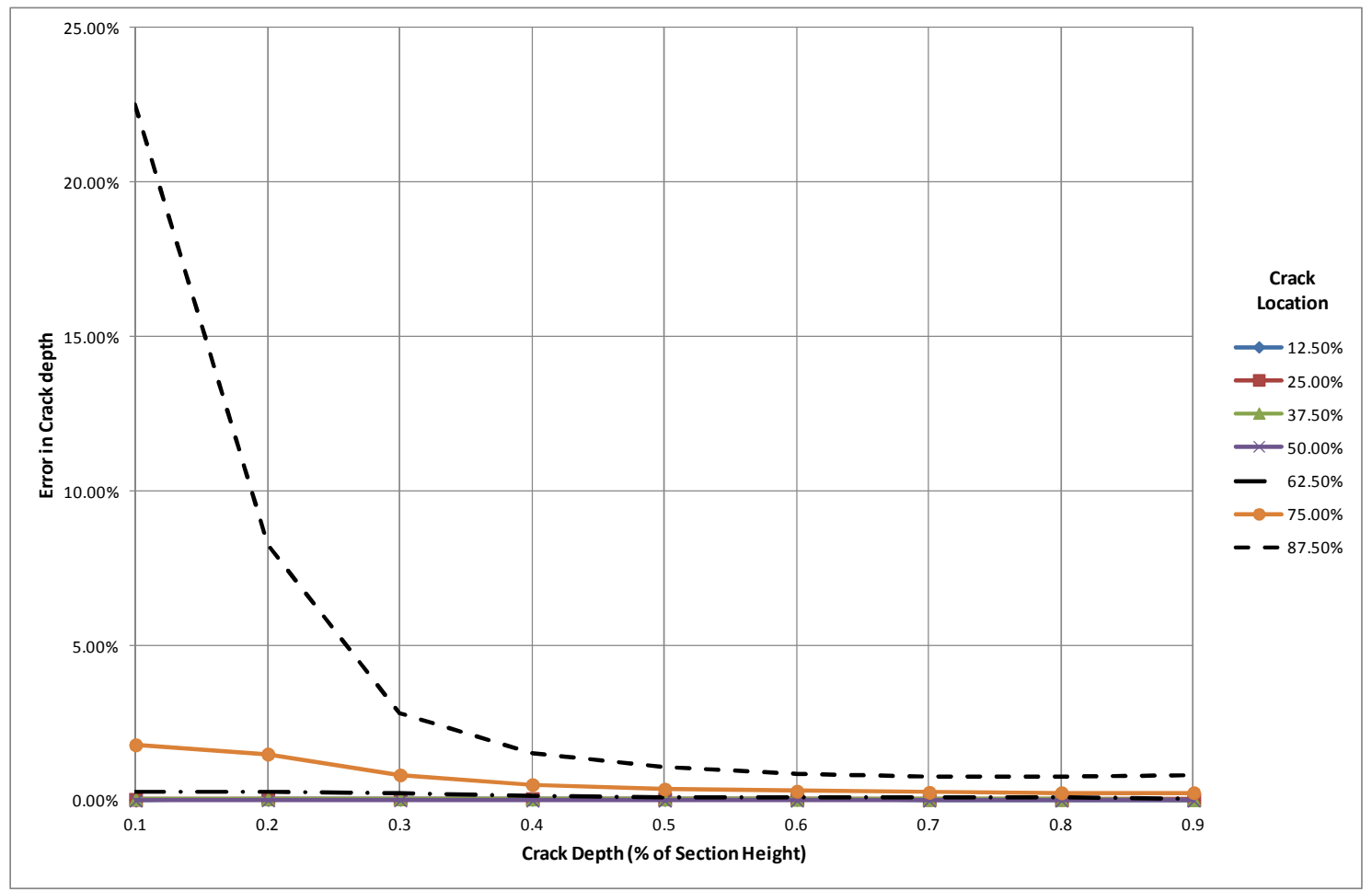

Fig 11. Pinned-Roller: Error in crack depth for different crack locations. 


\section{APPENDIX}

The boundary and compatibility conditions caste in matrix form for cracked cantilever (Fixed-Free) beam:

$\left[\begin{array}{cccccccc}1 & 0 & 1 & 0 & 0 & 0 & 0 & 0 \\ 0 & 1 & 0 & 1 & 0 & 0 & 0 & 0 \\ C(\beta) & S(\beta) & C h(\beta) & S h(\beta) & -C(\beta) & -S(\beta) & -C h(\beta) & -S h(\beta) \\ -C(\beta) & -S(\beta) & C h(\beta) & S h(\beta) & C(\beta) & S(\beta) & -C h(\beta) & -S h(\beta) \\ S(\beta) & -C(\beta) & S h(\beta) & C h(\beta) & -S(\beta) & C(\beta) & -S h(\beta) & -C h(\beta) \\ \lambda C(\beta)+\frac{K_{r}}{E I} S(\beta) & \lambda S(\beta)-\frac{K_{r}}{E I} C(\beta) & -\lambda C h(\beta)-\frac{K_{r}}{E I} \operatorname{Sh}(\beta) & -\lambda S h(\beta)-\frac{K_{r}}{E I} \operatorname{Ch}(\beta) & \frac{-K_{r}}{E I} S(\beta) & \frac{K_{r}}{E I} C(\beta) & \frac{K_{r}}{E I} S h(\beta) & \frac{K_{r}}{E I} \operatorname{Ch}(\beta) \\ 0 & 0 & 0 & 0 & -C(\lambda L) & -S(\lambda L) & C h(\lambda L) & S h(\lambda L) \\ 0 & 0 & 0 & 0 & S(\lambda L) & -C(\lambda L) & S h(\lambda L) & C h(\lambda L)\end{array}\right]\left\{\begin{array}{l}A_{1} \\ B_{1} \\ C_{1} \\ D_{1} \\ A_{2} \\ B_{2} \\ C_{2} \\ D_{2}\end{array}\right\}=0$

The boundary and compatibility conditions cast in matrix form for cracked simply supported (Pinned-Pinned and Pinned-Roller) beams:

$\left[\begin{array}{cccccccc}1 & 0 & 1 & 0 & 0 & 0 & 0 & 0 \\ -1 & 0 & 1 & 0 & 0 & 0 & 0 & 0 \\ C(\beta) & S(\beta) & C h(\beta) & S h(\beta) & -C(\beta) & -S(\beta) & -C h(\beta) & -S h(\beta) \\ -C(\beta) & -S(\beta) & C h(\beta) & S h(\beta) & C(\beta) & S(\beta) & -C h(\beta) & -S h(\beta) \\ S(\beta) & -C(\beta) & S h(\beta) & C h(\beta) & -S(\beta) & C(\beta) & -S h(\beta) & -C h(\beta) \\ \lambda C(\beta)+\frac{K_{r}}{E I} S(\beta) & \lambda S(\beta)-\frac{K_{r}}{E I} C(\beta) & -\lambda C h(\beta)-\frac{K_{r}}{E I} \operatorname{Sh}(\beta) & -\lambda S h(\beta)-\frac{K_{r}}{E I} C h(\beta) & \frac{-K_{r}}{E I} S(\beta) & \frac{K_{r}}{E I} C(\beta) & \frac{K_{r}}{E I} S h(\beta) & \frac{K_{r}}{E I} \operatorname{Ch}(\beta) \\ 0 & 0 & 0 & 0 & C(\lambda L) & S(\lambda L) & C h(\lambda L) & S h(\lambda L) \\ 0 & 0 & 0 & 0 & -C(\lambda L) & -S(\lambda L) & C h(\lambda L) & S h(\lambda L)\end{array}\right]\left\{\begin{array}{l}A_{1} \\ B_{1} \\ C_{1} \\ D_{1} \\ A_{2} \\ B_{2} \\ C_{2} \\ D_{2}\end{array}\right\}=0$

The boundary and compatibility conditions cast in matrix form for cracked FixedPinned and Fixed-Roller beams:

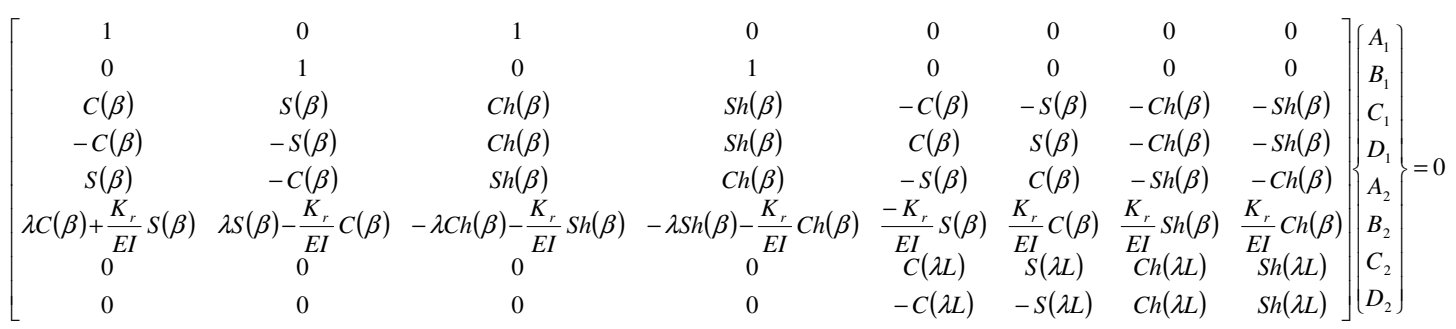

